\title{
Fragmentation Functions beyond Next-To-Leading Order Accuracy
}

\section{Daniele Paolo Anderle*}

Institute for Theoretical Physics, University of Tübingen, Germany

E-mail: daniele-paolo.anderle@uni-tuebingen.de

\section{Tom Kaufmann}

Institute for Theoretical Physics, University of Tübingen, Germany

\section{Felix Ringer}

Los Alamos National Laboratory, Los Alamos, NM, USA

\section{Marco Stratmann}

Institute for Theoretical Physics, University of Tübingen, Germany

\begin{abstract}
With the ever increasing amount of precise data available for hadron production processes, the perturbative QCD framework is being extended to explore effects and corrections that go beyond the next-to-leading order accuracy. Fixed order calculations at next-to-next-to-leading order accuracy are required for a precise and reliable comparison to experimental results, and, consequently, the analyses of the non-perturbative structure of hadrons has to match this level of accuracy. Moreover, potentially important effects specific to some kinematical regions, such as the small- $z$ and large- $z$ regions in semi-inclusive $e^{+} e^{-}$annihilation, can be investigated through the means of all-order resummation techniques. In this talk we present a first analysis of parton-to-pion fragmentation functions at next-to-next-to-leading order accuracy based on single-inclusive pion production in electron-positron annihilation. All-order resummations of large logarithmic contributions at small- $z$ are included and their numerical impact is investigated.
\end{abstract}

XXIV International Workshop on Deep-Inelastic Scattering and Related Subjects

11-15 April, 2016

DESY Hamburg, Germany

\footnotetext{
* Speaker.
} 


\section{SIA hadron multiplicities and the time-like evolution}

We consider single inclusive annihilation (SIA) $e^{+} e^{-} \rightarrow \gamma / Z \rightarrow h X$, more specifically, hadron multiplicities defined as

$$
\frac{1}{\sigma_{\mathrm{tot}}} \frac{d \sigma^{h}}{d z}=\frac{1}{\sigma_{\mathrm{tot}}} \sum_{k=T, L} \frac{d \sigma_{k}^{h}}{d z},
$$

with the total cross section $\sigma_{\text {tot }}[1,2]$. The parity-violating interference term of vector and axialvector contributions, usually called asymmetric $(A)$, is not present as we have already integrated over the scattering angle $\theta$. Hence, only a transverse $(T)$ and a longitudinal $(L)$ part remains. Furthermore, we have introduced the scaling variable $z \equiv 2 P_{h} \cdot q / Q^{2}$, where $P_{h}$ and $q$ are the four momenta of the observed hadron and $\gamma / Z$ boson, respectively. Moreover, $Q^{2} \equiv q^{2}=S$ with $\sqrt{S}$ being the c.m.s. energy. The transverse and longitudinal cross sections in Eq. (1.1) may be written in a factorized form as

$$
\begin{aligned}
\frac{d \sigma_{k}^{h}}{d z}=\sigma_{\mathrm{tot}}^{(0)} & {\left[D_{S}^{h}\left(z, \mu^{2}\right) \otimes \mathbb{C}_{k, q}^{\mathrm{S}}\left(z, \frac{Q^{2}}{\mu^{2}}\right)+D_{g}^{h}\left(z, \mu^{2}\right) \otimes \mathbb{C}_{k, g}^{\mathrm{S}}\left(z, \frac{Q^{2}}{\mu^{2}}\right)\right] } \\
& +\sum_{q} \sigma_{q}^{(0)} D_{\mathrm{NS}, q}^{h}\left(z, \mu^{2}\right) \otimes \mathbb{C}_{k, q}^{\mathrm{NS}}\left(z, \frac{Q^{2}}{\mu^{2}}\right) .
\end{aligned}
$$

For simplicity, we have chosen $\mu_{R}=\mu_{F} \equiv \mu$. Moreover, $\sigma_{q}^{(0)}=3 \sigma_{0} \hat{e}_{q}^{2}$ is the total quark production cross section for a given flavor $q$ at leading-order (LO), where $\sigma_{0}=4 \pi \alpha^{2} /\left(3 Q^{2}\right)$ is the LO QED cross section for $e^{+} e^{-} \rightarrow \mu^{+} \mu^{-}$and $\alpha$ denotes the electromagnetic coupling constant. The electroweak quark charges $\hat{e}_{q}$ can be found, e.g., in Ref. [1]. We also have defined $\sigma_{\text {tot }}^{(0)}=\sum_{q} \sigma_{q}^{(0)}$. The symbol $\otimes$ denotes the standard convolution integral defined as

$$
f(z) \otimes g(z) \equiv \int_{0}^{1} d x \int_{0}^{1} d y f(x) g(y) \delta(z-x y) .
$$

As usual, the factorized structure of Eq. (1.2) only holds in the presence of a hard scale. In our case, the hard scale is given by $Q$. The non-perturbative but universal fragmentation functions (FFs) $D_{i}^{h}\left(z, \mu^{2}\right)$ parametrize the formation of a hadron $h$ from a parton, $i=q, \bar{q}, g$. Both the parton and the hadron are assumed to be massless. The argument $z$ is the longitudinal momentum fraction transferred to the hadron. The quark singlet (S) and non-singlet (NS) combinations appearing in Eq. (1.2) are defined as

$$
D_{S}^{h}\left(z, \mu^{2}\right)=\frac{1}{N_{f}} \sum_{q}\left[D_{q}^{h}\left(z, \mu^{2}\right)+D_{\bar{q}}^{h}\left(z, \mu^{2}\right)\right], \quad D_{\mathrm{NS}, q}^{h}\left(z, \mu^{2}\right)=D_{q}^{h}\left(z, \mu^{2}\right)+D_{\bar{q}}^{h}\left(z, \mu^{2}\right)-D_{\mathrm{S}}^{h}\left(z, \mu^{2}\right)
$$

respectively. The corresponding $i=\mathrm{S}$, NS coefficient functions can be calculated as a perturbative series in $a_{s} \equiv \alpha_{s} / 4 \pi$,

$$
\mathbb{C}_{k, l}^{i}=\mathbb{C}_{k, l}^{i,(0)}+a_{s} \mathbb{C}_{k, l}^{i,(1)}+a_{s}^{2} \mathbb{C}_{k, l}^{i,(2)}+\mathscr{O}\left(a_{s}^{3}\right),
$$

where the arguments $\left(z, Q^{2} / \mu^{2}\right)$ have been suppressed. Results are available up to $\mathscr{O}\left(a_{s}^{2}\right)[1,3,4]$ which is next-to-next-to-leading-order (NNLO) for the transverse but formally only of next-toleading order (NLO) accuracy for the longitudinal coefficient functions. These fixed order calculations show logarithmic divergencies of the coefficient functions for $z \rightarrow 1$ (large- $z$ regime) and 
$z \rightarrow 0$ (small-z). Focusing only on the small- $z$ regime, one finds a double logarithmic enhancement. For example, for the gluon sector

$$
\mathbb{C}_{T, g}^{S,(k)} \propto a_{s}^{k} \frac{1}{z} \log ^{2 k-1-a}(z), \quad \mathbb{C}_{L, g}^{S,(k)} \propto a_{s}^{k} \frac{1}{z} \log ^{2 k-2-a}(z),
$$

with $a=0,1,2$ identifying the leading-logarithmic (LL), next-to-leading-logarithmic (NLL) and next-to-next-to-leading-logarithmic (NNLL) contribution respectively. Furthermore, the same logarithmic behaviour for small- $z$ is shown in the time-like splitting functions appearing in the evolution of the FFs, e.g. for the gluon-to-gluon or quark-to-gluon splitting function we find

$$
P_{g i}^{T,(k)} \propto a_{s}^{(k+1)} \frac{1}{z} \log ^{2 k-a}(z),
$$

where $i=q, g$. As it is well established, such divergent terms may spoil perturbation theory even for very small values of the strong coupling constant $a_{s} \ll 1$. The usual approach is to take into account those logarithms to all orders via resummation techniques. Resummed expressions for both splitting and coefficient functions in Mellin $N$ space are given up to NNLL accuracy in the literature $[9,10]$. In general, the Mellin transform $f(N)$ of a function $f(z)$ is defined by

$$
f(N)=\int_{0}^{1} d z z^{N-1} f(z) \equiv \mathscr{M}[f(z)] .
$$

The logarithms that appear in Eqs. (1.6) and (1.7) correspond to singularities at $\bar{N} \equiv N-1=0$,

$$
\mathscr{M}\left[\frac{\log ^{2 k-1}(z)}{z}\right]=(-1)^{k} \frac{(2 k-1) !}{\bar{N}^{2 k}} .
$$

Analogously to the case of parton distributions functions, the dependence on the factorization scale $\mu$ of FFs (1.4) may be calculated in pQCD by solving a set of $2 N_{f}+1$ RGEs, which correspond to a time-like version of the DGLAP evolution equations [5]. In Ref. [6] a detailed review on how to numerically implement the fixed order solution at NNLO accuracy is given. It follows closely the framework and the strategies developed for the public space-like evolution code PEGASUS [7], and it makes use of the Mellin space results for the NNLO splitting functions [8]. In Mellin space the general analytical solution to the time-like DGLAP equations, can be written in terms of the so called evolution matrices $U_{k}$ as

$$
D^{h}\left(N, a_{s}(\mu)\right)=\left[1+\sum_{k=1}^{\infty} a_{s}^{k} U_{k}(N)\right]\left(\frac{a_{s}(\mu)}{a_{s}\left(\mu_{0}\right)}\right)^{-R_{0}(N)}\left[1+\sum_{k=1}^{\infty} a_{s}^{k} U_{k}(N)\right]^{-1} D^{h}\left(N, a_{s}\left(\mu_{0}\right)\right)
$$

The $U_{k}$ are recursively defined by the commutation relations

$$
\left[U_{k}, R_{0}\right]=R_{k}+\sum_{i=1}^{k-1} R_{k-1} U_{i}+k U_{k}
$$

In Eq. $1.10, D^{h}$ is either the singlet vector $\left(D_{S}^{h}, D_{g}^{h}\right)$ or one of the non-singlet FFs' combinations. Correspondently, $U_{k}$ and $R_{k}$ are either $2 \times 2$ matrices or scalar valued functions of $N$. The $R_{k}$ in (1.11) are defined recursively as

$$
R_{0} \equiv \frac{1}{\beta_{0}} P^{T,(0)}, R_{k} \equiv \frac{1}{\beta_{0}} P^{T,(k)}-\sum_{i=1}^{k} b_{i} R_{k-i}
$$


where in case of $R_{k}$ being matricies, $P^{T,(k)}$ represent the matrices with entries $P_{i j}^{T,(k)}(i, j=q, g)$, otherwise they represent the appropriate non-singlet splitting function. In addition, $b_{i} \equiv \beta_{i} / \beta_{0}$ with $\beta_{k}$ denoting the expansion coefficients of the QCD $\beta$-function. Based on (1.10), it is possible to define several solutions at order $\mathrm{N}^{\mathrm{m}} \mathrm{LO}$ which are all equivalent up to the accuracy considered, i.e., up to subleading higher-order terms. Given the iterative definition of the $R_{k}$ in Eq. (1.12), one possibility is to calculate the $R_{k}$ and, hence the $U_{k}$ in Eq. (1.11), for any $k>m$ from the known results for $P^{T,(k)}$ and $\beta_{k}$ up to $k=m$. Any higher order $P^{T,(k)}$ and $\beta_{k}$ with $k>m$ are simply set to zero. Taking into account all the thus constructed $U_{k}$ in Eq. (1.10) defines the so-called iterated solution. To include in the evolution the small- $z$ logarithmic contributions appearing at all orders in the splitting functions, we start by considering the expansion of the fully resummed expressions $[9,10], P_{j l}^{T \mathrm{~N}^{\kappa} \mathrm{LL}}=\sum_{n=0}^{\infty} a_{s}^{n+1} P_{j l}^{T \mathrm{~N}^{\kappa} \mathrm{LL},(n)}$, where the $P_{j l}^{T \mathrm{~N}^{\kappa} \mathrm{LL},(n)}$ are known up to NNLL accuracy, i.e., for $\kappa=0,1,2$. The simplest way of extending the framework of [6] to the resummed case is to utilize the iterated solution and set $P^{T,(k)} \equiv P^{T \mathrm{~N}^{k} \mathrm{LL},(k)}$ for every $k$, including terms with $k>m$ in Eq. (1.12). The number $k$ of $P^{T \mathrm{~N}^{k} \mathrm{LL},(k)}$ needed for the relevant $z$ range in the iterated solution can be asses by studying the convergence of the series expansion of the resummed expressions as shown in Fig. 1a. A numerical Mellin inversion was used to compare the expanded with the resummed splitting functions in $z$ space. We found that $k=20$ is pretty accurate (less than $0.3 \%$ difference) down to values of $z \approx 10^{-5}$ which is more than sufficient to study the data sets available at the time of writing.

\section{Fit to SIA data}

To asses the relevance of both NNLO and small- $z$ logarithmic contributions we perform a fit to SIA data with identified pions, more specifically, we use the same data sets as in [6]. For the fitting procedure, we adopt the same flexible functional form as in Ref. [6, 11]

$$
D_{i}^{\pi^{+}}\left(z, \mu_{0}^{2}\right)=\frac{N_{i} z^{\alpha_{i}}(1-z)^{\beta_{i}}\left[1+\gamma_{i}(1-z)^{\delta_{i}}\right]}{B\left[2+\alpha_{i}, \beta_{i}+1\right]+\gamma_{i} B\left[2+\alpha_{i}, \beta_{i}+\delta_{i}+1\right]}
$$

to parametrize the non-perturbative input FFs for charged pions at a scale $\mu_{0}$ in the $\overline{\mathrm{MS}}$ scheme. For this analysis, however, we set the initial scale $\mu_{0}=10.54 \mathrm{GeV}$, which is equivalent to the BABAR c.m.s. energy squared. $B[a, b]$ is the Euler Beta function used to normalize the parameter $N_{i}$ in (2.1) for each flavor $i$ to its contribution to the energy-momentum sum rule. In addition to the gluon $i=g$, we only consider FFs for the sum of a quark and an antiquark of a given flavor $i$, since SIA is only sensitive to $q+\bar{q}$ flavor combinations as can be already inferred from Eq. (1.2). In this analysis, we impose a global cut $0.01<z<0.95$ for all data points except for the TPC data where the lower cut is set to $z>0.02$. The total amount of data points taken into account in this analysis is 436 . Furthermore, we set $\gamma_{g, s+\bar{s}, c+\bar{c}}=0$ in Eq. (2.1). Thus, we are left with 19 free parameters in our fit. We are most interested in the comparison of fixed order results with the resummed ones. This allows us to quantify the relevance of small- $z$ resummations in FF analyses. As usual the matching of a resummed observable $T^{\text {res }}$ to its $\mathrm{N}^{\kappa} \mathrm{LO}$ fixed order expression $T^{\mathrm{N}^{\kappa} \mathrm{LO}}$ is performed according to $T^{\text {matched }}=T^{\mathrm{N}^{\kappa} \mathrm{LO}}+T^{\text {res }}-\left.T^{\mathrm{res}}\right|_{\mathscr{O}\left(a_{s}^{\kappa}\right)}$, where $\left.T^{\text {res }}\right|_{\mathscr{O}\left(a_{s}^{\kappa}\right)}$ denotes the expansion of $T^{\text {res }}$ up to order $\mathscr{O}\left(a_{s}^{\kappa}\right)$. We choose the logarithmic order in such a way that we do not resum logarithmic contributions which are not present in the fixed order result. For this reason, we match the LO calculation 
Table 1: The $\chi^{2}$-values and the $\chi^{2}$ per degree of freedom (d.o.f) for our fits at fixed order and with resummations included.

\begin{tabular}{lccllcc} 
accuracy & $\chi^{2}$ & $\chi^{2} /$ d.o.f. & & accuracy & $\chi^{2}$ & $\chi^{2} /$ d.o.f. \\
\hline LO & 1260.8 & 2.89 & & LO+LL & 405.5 & 0.93 \\
NLO & 354.1 & 0.81 & & NLO+NNLL & 352.3 & 0.81 \\
NNLO & 330.1 & 0.76 & & NNLO+NNLL & 330.0 & 0.76
\end{tabular}

only with LL as the only logarithmic contribution showing up at LO is of LL accuracy. Following the same philosophy, we match NLO together with the NNLL results. Finally, at NNLO five powers of small- $z$ logarithms are present. However, the most accurate resummed result currently available is at NNLL accuracy. Thus, we have to match NNLO only with NNLL due to the missing higher order expressions. The resulting $\chi^{2}$-values and the $\chi^{2}$ per degree of freedom are shown in Tab. 1. The main features of the considered corrections can be read off directly from said Table: a LO fit is not able to describe the experimental results adequately. A NLO fit already yields an acceptable result, which is further improved upon including NNLO corrections. Compared to the corresponding fixed order results, the fits including also all-order resummations of small- $z$ logarithms exhibit only a slighly better $\chi^{2}$, except for LO+LL where resummations lead to a significant improvement in the quality of the fit. Differences between fits at NNLO and NNLO+NNLL accuracy are not significant. Hence, we conclude that in the $z$-range where experimental results are available, NNLO expressions already capture all the relevant features to yield a satisfactory fit to data and small- $z$ resummations are not really necessary phenomenologically.

In Fig. 1b we plot the FFs resulting from our fits. We show the gluon and singlet FFs, $D_{g}^{\pi^{+}}\left(z, Q^{2}\right)$ and $D_{\Sigma}^{\pi^{+}}\left(z, Q^{2}\right) \equiv N_{f} D_{S}^{\pi^{+}}\left(z, Q^{2}\right)$, respectively, for the LEP c.m.s. energy, i.e., $Q=91.2$ $\mathrm{GeV}$. It is worth noticing that the resummed expressions for which we have full control over all logarithmic powers (i.e. LO+LL and NLO+NNLL) are well behaved at small- $z$ and show the expected oscillatory behavior which they inherit from the Bessel function's shape of the splitting functions through the mean of the evolution (see [10]). The NNLO+NNLL FF still goes to minus infinity, as we do not have control over all five logarithmic powers appearing at fixed order. However, because we have resummed the three leading towers, it falls much softer compared to NNLO.

\section{Conclusions}

With the presented, new SIA analysis, we extended the results of [6] to include all-order resummation for the small- $z$ logarithmic contributions. In order to do so, we made use of the resummed expressions for the time-like splitting and coefficient functions given in Refs. [9, 10]. A first phenomenological study was performed to estimate the numerical impact of those contributions in fits of pion fragmentation functions in the kinematical $z$-range where experimental data are available. It turns out that a fixed order analysis at NNLO accuracy appears to be sufficient to reliably extract FFs from the data available. Although resummation effects cannot be ignored from a theoretical point of view, the small- $z$ behavior in the phenomenologically relevant kinematical region is already well described by terms appearing in the fixed order expressions at NNLO accuracy. 


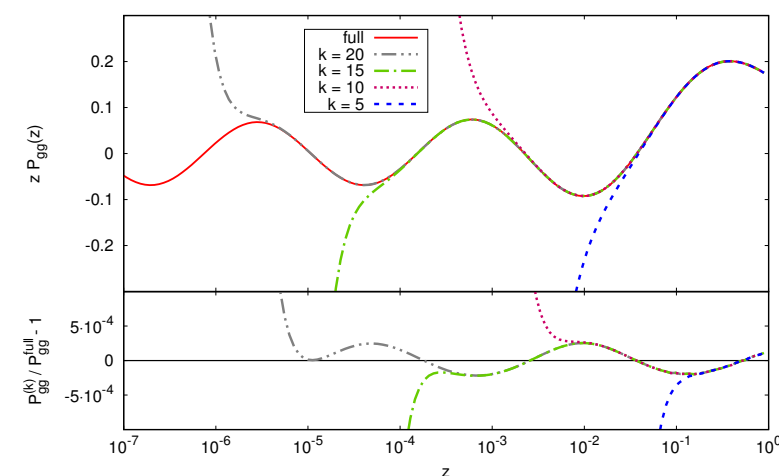

a)

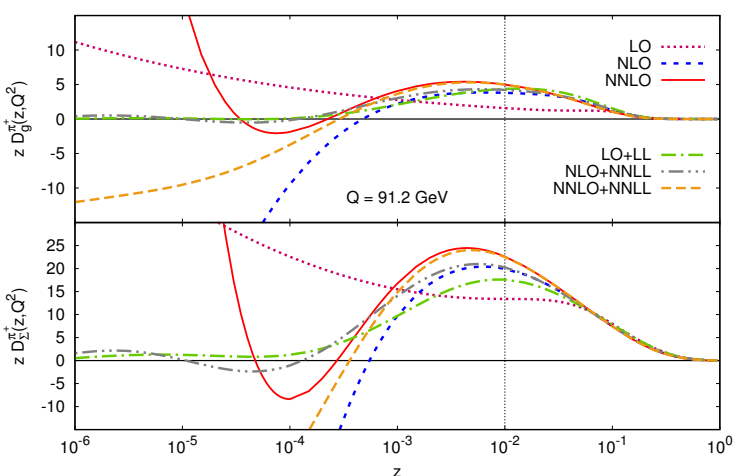

b)

Figure 1: a) $z$ times the splitting function $P_{g g}(z)$ at NNLL for different maximum values of $k$ compared to the full resummed expression. In the lower panel we show the deviation of the expanded splitting function compared to the full one. Everything is evaluated for $Q^{2}=110 \mathrm{GeV}^{2}$ and $N_{f}=5$. b) Our gluon and singlet FFs at LEP energy $(Q=91.2 \mathrm{GeV})$ obtained from the fits listed in Tab. 1 . The singlet is shown for $N_{f}=5$. The dotted vertical line at $z=0.01$ shows the cutoff in our fit.

\section{Acknowledgments}

We are grateful to W. Vogelsang, and A. Vogt for helpful discussions and comments. D.P.A. acknowledges partial support from the Fondazione Cassa Rurale di Trento. This work was supported in part by the Deutsche Forschungsgemeinschaft (DFG) grant VO 1049/1 and ZUK 63, by the Bundesministerium für Bildung und Forschung (BMBF) Grant No. 05P15VTCA1 and the U.S. Department of Energy.

\section{References}

[1] P. J. Rijken and W. L. van Neerven, Nucl. Phys. B 487, 233 (1997).

[2] K. G. Chetyrkin, A. L. Kataev, and F. V. Tkachov, Phys. Lett. B 85, 277 (1979); M. Dine and J. Sapirstein, Phys. Rev. Lett. 43, 668 (1979); W. Celmaster and R. J. Gonsalves, Phys. Rev. Lett. 44, 560 (1980).

[3] P. J. Rijken and W. L. van Neerven, Phys. Lett. B 386, 422 (1996).

[4] A. Mitov and S. O. Moch, Nucl. Phys. B 751, 18 (2006).

[5] V. N. Gribov and L. N. Lipatov, Sov. J. Nucl. Phys. 15, 438 (1972); L. N. Lipatov, Sov. J. Nucl. Phys. 20, 94 (1975); G. Altarelli and G. Parisi, Nucl. Phys. B 126, 298 (1977); Y. L. Dokshitzer, Sov. Phys. JETP 46, 641 (1977).

[6] D. P. Anderle, F. Ringer and M. Stratmann, Phys. Rev. D 92, 114017 (2015).

[7] A. Vogt, Comput. Phys. Commun. 170, 65 (2005).

[8] A. Mitov, S. Moch, and A. Vogt, Phys. Lett. B 638, 61 (2006); S. Moch and A. Vogt, Phys. Lett. B 659, 290 (2008); A. A. Almasy, S. Moch, and A. Vogt, Nucl. Phys. B 854, 133 (2012).

[9] A. Vogt, JHEP 1110, 025 (2011).

[10] C.-H. Kom, A. Vogt and K. Yeats, JHEP 1210, 033 (2012).

[11] D. de Florian, R. Sassot, and M. Stratmann, Phys. Rev. D 75, 114010 (2007); Phys. Rev. D 76, 074033 (2007); D. de Florian, R. Sassot, M. Epele, R. J. Hernandez-Pinto, and M. Stratmann, Phys. Rev. D 91, 014035 (2015). 